\title{
Correlation between the Relative Sensitivity Factors and the Sputtering Yields in Glow-Discharge Mass Spectrometry
}

\author{
Takashi SAKA ${ }^{*, \dagger}$ and Mika INOUE** \\ *Department of Applied Electronics, Daido Institute of Technology, 2-21 Daido-cho, Minami, Nagoya \\ 457-8531, Japan \\ **Research and Development Lab., Daido Steel Co. Ltd., 2-30 Daido-cho, Minami, Nagoya 457-8545, Japan
}

(Received January 14, 2000; Accepted March 21, 2000)

In trace elemental analyses by glow-discharge mass spectrometry (GDMS), samples are sputtered by plasma ions in a glow discharge, and sputtered neutral atoms are subsequently ionized in the plasma. The ions are introduced into a mass spectrometer and the ion-beam intensities of analyzed elements are measured with reference to a matrix element; the ratios of these ion beam intensities are obtained. ${ }^{1,2}$ The concentration of an analyzed element is determined by multiplying the observed ion-beam ratio by a relative sensitivity factor (RSF). RSFs are related to the ratios of the ion-beam intensities by the following definition: ${ }^{2}$

$$
\operatorname{RSF}_{\mathrm{X}}=\left(C_{\mathrm{X}} / C_{\mathrm{R}}\right) /\left(I_{\mathrm{X}} / I_{\mathrm{R}}\right),
$$

where suffices $\mathrm{X}$ and $\mathrm{R}$ indicate that the quantities are relevant to an analyzed element and a reference (matrix) element, respectively, and $\mathrm{I}$ and $\mathrm{C}$ are the ion beam intensities and the concentrations by weight, respectively. In GDMS analyses, because the quantitative accuracy is seriously affected by the accuracy of RSFs used, much work has been carried out to obtain accurate RSFs of many elements in many matrices. ${ }^{3-5}$ At the same time, investigations to clarify the effects of many factors, such as the discharge conditions, sample shapes or matrices on RSFs, have been carried out and theoretical investigations to estimate RSFs are also in progress. ${ }^{5-14}$ However, there still exist disagreements between the observed RSFs and the calculated ones. Hitherto, both the experimental and the theoretical efforts have mainly been focussed on the ionization processes in a glow discharge. On the other hand, we have already pointed out that there exists a correlation between the RSFs and the sputtering yields. ${ }^{5}$ It has a possibility to shed light on our understanding of RSFs to take the sputtering process of samples into consideration. In this paper the correlation between RSFs and sputtering yields is reported in more detail.

\section{Experimental}

RSFs have already been determined using pin-shaped samples and disk-shaped samples. ${ }^{5}$ For the details concerning the samples and the experimental conditions, the previous paper should be referred to, and only some essential points are summarized here. Samples available commercially were used, the matrices being

† To whom correspondence should be addressed.

E-mail: saka@daido-it.ac.jp
$\mathrm{Fe}, \mathrm{Ni}, \mathrm{Cu}, \mathrm{Al}$ and Ti. For the Fe- and Ni-based metals, a few samples cast at Daido Steel Co. were also used. The ion-beam

Table 1 Numerical list of RSFs with the reference to Fe

\begin{tabular}{|c|c|c|}
\hline \multirow{2}{*}{ Element } & \multicolumn{2}{|c|}{ RSF } \\
\hline & Pin & Disk \\
\hline $\mathrm{Be}$ & 1.38 & 1.07 \\
\hline $\mathrm{B}$ & 1.35 & 1.63 \\
\hline $\mathrm{C}$ & 4.67 & 3.60 \\
\hline $\mathrm{Mg}$ & 1.49 & 1.33 \\
\hline $\mathrm{Al}$ & 0.91 & 0.99 \\
\hline $\mathrm{Si}$ & 1.71 & 1.65 \\
\hline $\mathrm{P}$ & 3.13 & 2.81 \\
\hline$S$ & 2.64 & 2.82 \\
\hline $\mathrm{Ca}$ & 0.37 & 0.46 \\
\hline $\mathrm{Ti}$ & 0.42 & 0.52 \\
\hline $\mathrm{V}$ & 0.56 & 0.64 \\
\hline $\mathrm{Cr}$ & 2.15 & 1.95 \\
\hline $\mathrm{Mn}$ & 1.55 & 1.48 \\
\hline $\mathrm{Fe}$ & 1.07 & 1.00 \\
\hline $\mathrm{Co}$ & 0.89 & 0.86 \\
\hline $\mathrm{Ni}$ & 1.14 & 1.20 \\
\hline $\mathrm{Cu}$ & 4.72 & 3.91 \\
\hline $\mathrm{Zn}$ & 5.22 & 4.08 \\
\hline $\mathrm{Ga}$ & 3.47 & 3.10 \\
\hline As & 5.08 & 3.82 \\
\hline $\mathrm{Se}$ & 2.72 & 2.41 \\
\hline $\mathrm{Zr}$ & 0.68 & 0.71 \\
\hline $\mathrm{Nb}$ & 0.60 & 0.73 \\
\hline Mo & 1.26 & 1.23 \\
\hline $\mathrm{Ag}$ & 3.90 & 3.68 \\
\hline $\mathrm{Cd}$ & 7.08 & 5.09 \\
\hline In & 3.89 & 3.53 \\
\hline $\mathrm{Sn}$ & 2.59 & 2.14 \\
\hline $\mathrm{Sb}$ & 4.47 & 3.50 \\
\hline $\mathrm{Te}$ & 4.25 & 3.43 \\
\hline $\mathrm{La}$ & 0.74 & 1.16 \\
\hline $\mathrm{Ce}$ & 0.63 & 1.12 \\
\hline $\operatorname{Pr}$ & 0.60 & 1.21 \\
\hline $\mathrm{Nd}$ & 0.60 & 0.96 \\
\hline $\mathrm{Ta}$ & 1.13 & 1.31 \\
\hline W & 1.73 & 1.48 \\
\hline $\mathrm{Au}$ & 3.24 & 3.11 \\
\hline $\mathrm{Hg}$ & 9.77 & 6.60 \\
\hline $\mathrm{Tl}$ & 7.98 & 6.03 \\
\hline $\mathrm{Pb}$ & 2.36 & 1.88 \\
\hline $\mathrm{Bi}$ & 4.52 & 3.33 \\
\hline
\end{tabular}




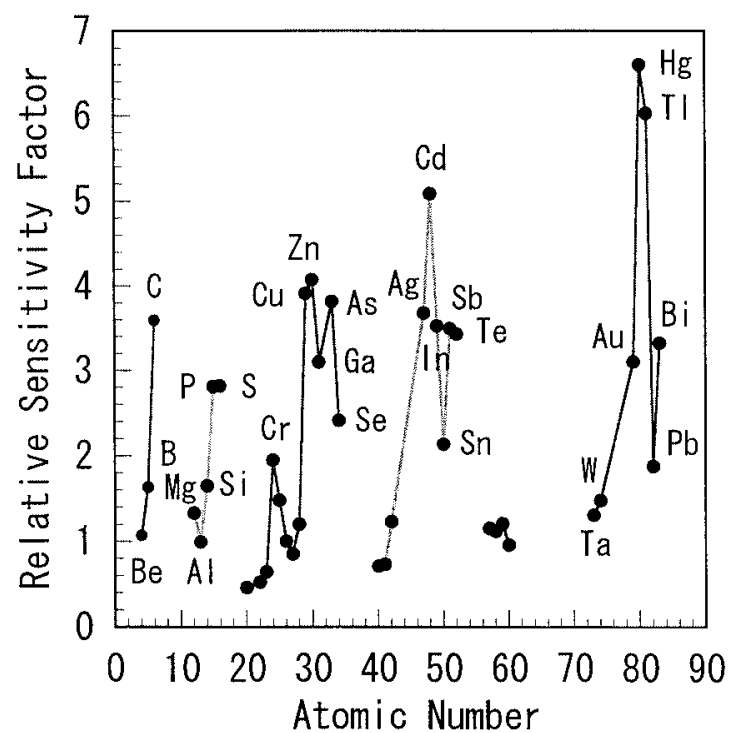

Fig. 1 Relation between the atomic number and the RSFs for diskshaped samples.

intensities for many elements as well as each matrix were measured and the RSFs with the reference to the respective matrices were determined by the gradients of calibration lines composed of $I_{\mathrm{X}} / I_{\mathrm{R}}$ and $C_{\mathrm{X}} / C_{\mathrm{R}}$, as defined by Eq. (1).

In the previous paper, RSFs were determined for each isotope of some elements. Here, the RSFs of the most abundant isotopes in the respective elements are employed as we focus our interests on the relations between the elements and the RSFs.

\section{Results and Discussion}

\section{Conversion of reference matrices into $\mathrm{Fe}$}

The RSFs with reference to the matrices other than $\mathrm{Fe}$ were converted so that these are referred to $\mathrm{Fe}$ by the following method. First, it was confirmed that simple proportional relations exist between the RSFs for the Fe matrix and those for each of other matrices. Second, using the method of least squares the proportionality factors between the RSFs for the Fe matrix and the other matrices were determined. Last, all of the RSFs for matrices other than Fe were multiplied by the relevant proportionality factors. By this method, the reference matrices were converted into $\mathrm{Fe}$. No systematic deviations were recognized among the five sets of the RSFs of the different matrices after this conversion of the matrices. Then, the averaged values were determined, which are regarded as the most probable RSFs. This procedure has been applied to both pin-shaped and disk-shaped samples. The results are given in Table 1. In Table 1 the RSF of $\mathrm{Fe}$ as a matrix deviates from unity for the pin-shaped samples, though $\mathrm{Fe}$ is the reference element. This is caused because the averaged values on the different matrices are employed. This deviation is not necessary to be modified, because only the relative values are important in the following discussion.

\section{Correlation between the RSFs and the sputtering yields}

In the present paper, the RSFs determined using disk-shaped samples are mainly discussed, because they are considered to be more accurate than those determined using pin-shaped samples. ${ }^{5}$ Figure 1 shows the dependence of the observed RSFs on the

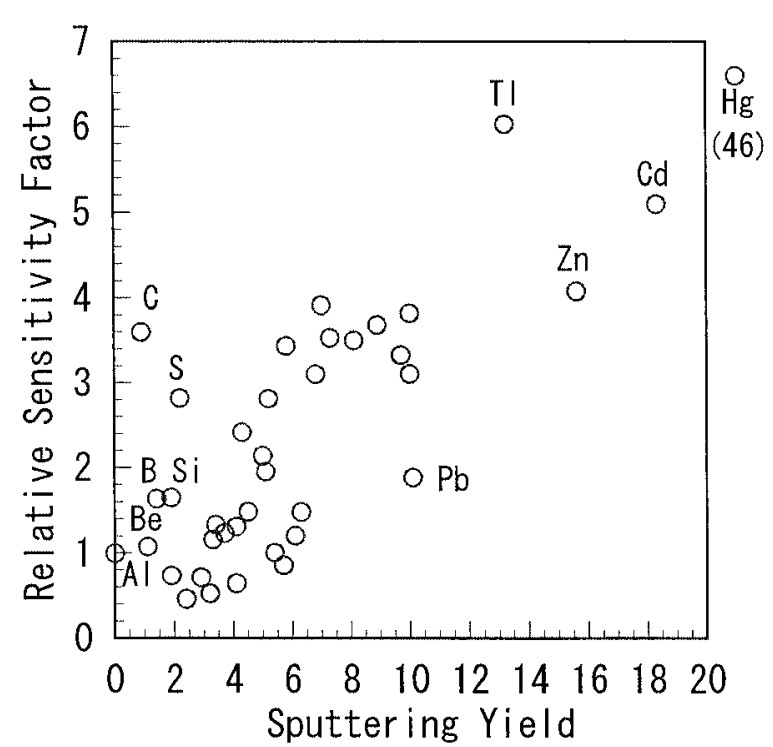

Fig. 2 Correlation of RSFs for disk-shaped samples with sputtering yields determined by Kanaya et al.

atomic number. A similar dependence on the atomic number has been recognized in sputtering yields determined by many authors. ${ }^{15-17}$ As an example, a presentation of the RSFs and the sputtering yields determined by Kanaya et al. ${ }^{15}$ is shown in Fig. 2 , where the sputtering yields represent the maximum sputtering yields measured using a projectile of Ar ions. Though some scatters are shown especially for light elements, there exists a clear correlation between these two quantities, the correlation coefficient being 0.73 . The same correlation has also been recognized between the RSFs and sputtering yields determined by Laegreid and Wehner, ${ }^{16}$ the projectile and the accelerating voltage being Ar ions and $0.6 \mathrm{kV}$, respectively. For simulated sputtering yields by Matsunami et al. ${ }^{17}$ the same result has been obtained. The correlation coefficients are 0.86 and 0.69 for the sputtering yields by Laegreid and Wehner and Matsunami et al., respectively. This correlation reveals that for elements that are easily sputtered, weak ion beams are observed in GDMS and vice versa.

\section{Dependence of the sputtering yields on the energy of projectiles}

The energy of projectiles just before bombardment on a sample in a glow-discharge mass spectrometer is much lower than the potential energy between the samples and the cell, and the probable energy of the bombardment will be $10-20 \%$ of the potential energy. ${ }^{18}$ In the present case the applied voltage was $800 \mathrm{~V}$. Therefore, the probable energy is about $80-160 \mathrm{eV}$, which is much smaller than those values used to determine the sputtering yields. Nevertheless, the behavior of the correlation is similar among the three sets of data with different projectile energies. This similarity is expected from the fact that the sputtering yields depend on the energy of projectiles in almost the same manner, especially for heavy atoms. ${ }^{17}$ However, the scatter in Fig. 2 for light elements, such as $\mathrm{C}$ and $\mathrm{S}$, are not explained by the energy dependence of the sputtering yields. The sputtering yields for such light elements bombarded with lower energies become much smaller than the maximum sputtering yields compared to the cases for heavier elements.

\section{Effects of sample shape on the correlation}

Hitherto, we have discussed the correlation for disk-shaped 


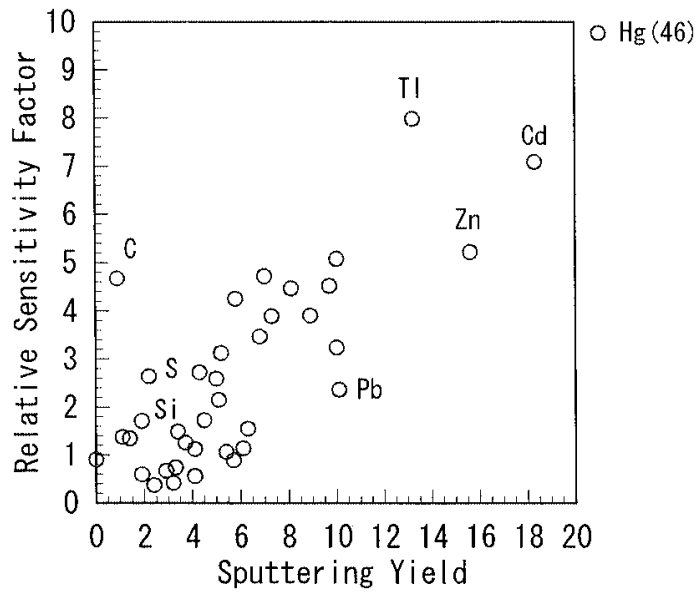

Fig. 3 Correlation of RSFs for pin-shaped samples with sputtering yields determined by Kanaya et al.

samples. The same correlation between the RSFs and the sputtering yields has also been observed for pin-shaped samples, as shown in Fig. 3, where the correlation coefficient is 0.79 . Therefore, the correlation will be present irrespective of the geometrical arrangements between a cell and a sample.

\section{Comparison with previous work}

The correlation shown in Figs. 2 and 3 indicates a possibility that there exist some processes concerned with sputtering affecting the RSFs in GDMS. This possibility is also inferred from the theoretical result by Vieth and Huneke. ${ }^{3}$ In their paper, a fairly good agreement has been obtained between the calculated RSFs and the observed ones. However, a large disagreement has been observed for elements of the IIB groups ( $\mathrm{Zn}, \mathrm{Cd}$ and $\mathrm{Hg}$ ), whose sputtering yields are very large. The most predominant processes influencing the RSFs are the Penning ionization process and an electron-impact process. However, some processes concerned with sputtering may exist, and investigations on these processes will be helpful for a clearer understanding of the RSFs.

\section{Acknowledgement}

One of the authors (T. S.) thanks Prof. J. Fujita of Daido Institute of Technology for his helpful discussions.

\section{References}

1. W. W. Harrison, K. R. Hess, R. K. Marcus, and F. L. King, Anal. Chem., 1986, 58, 341A.

2. N. E. Sanderson, E. Hall, J. Clark, P. Charalambous, and D. Hall, Mikrochim. Acta, 1987, 1, 275.

3. W. Vieth and J. C. Huneke, Spectrochim. Acta, 1991, 46B, 137.

4. A. P. Mykytiuk, P. Semeniuk, and S. Berman, Spectrochim. Acta Rev., 1990, 13, 1.

5. T. Saka, M. Inoue, and N. Okamoto, in Proceedings of the Fifth International Conference on Progress in Analytical Chemistry in the Steel and Metals Industries, ed. R. Tomellini, 1999, CETAS, Luxembourg , 579 - 586.

6. P. M. Charalambous, Steel Res., 1987, 58, 197.

7. K. Robinson and E. F. H. Hall, J. Metals, 1987, 39, 14.

8. N. Jakubowski and D. Stuewer, Fresenius Z. Anal. Chem., 1989, 335, 680 .

9. W. Vieth and J. C. Huneke, Spectrochim. Acta, 1991, 45B, 941.

10. S. Itoh, F. Hirose, and R. Hasegawa, Spectrochim. Acta, 1992, 47, 1241.

11. M. Saito, Anal. Chim. Acta, 1993, 274, 327.

12. M. Saito, Spectrochim. Acta, 1995, 50B, 171.

13. M. Saito, Fresenius J. Anal. Chem., 1995, 351, 148.

14. M. Saito, Fresenius J. Anal. Chem., 1997, 357, 18.

15. K. Kanaya, K. Hojou, K. Adachi, and K. Toki, Micron, $\mathbf{1 9 7 4}, 5,89$.

16. N. Laegreid and G. K. Wehner, J. Appl. Phys., 1961, 32, 365.

17. N. Matsunami, Y. Yamamura, Y. Itikawa, N. Itoh, Y. Kazumata, S. Miyagawa, K. Morita, R. Shimizu, and H. Tawara, Atomic Data and Nucl. Data Tables, 1984, 31, 1.

18. W. D. Davis and T. A. Vanderslice, Phys. Rev., 1963, 131, 219. 